\title{
ON WEAKLY COMPACT OPERATORS ON BANACH LATTICES
}

\author{
C. D. ALIPRANTIS AND O. BURKINSHAW
}

\begin{abstract}
Consider a Banach lattice $E$ and an order bounded weakly compact operator $T: E \rightarrow E$. The purpose of this note is to study the weak compactness of operators that are related with $T$ in some order sense. The main results are the following.

(1) If $T$ is a positive weakly compact operator and an operator $S: E \rightarrow E$ satisfies $0<S<T$, then $S^{2}$ is weakly compact. (Examples show that $S$ need not be weakly compact.)

(2) If $T$ and $S$ are as in (1) and either $S$ is an orthomorphism or $E$ has an order continuous norm, then $S$ is weakly compact.

(3) If $E$ is an abstract $L$-space and $T$ is weakly compact, then the modulus $|T|$ is weakly compact.
\end{abstract}

For notation and terminology concerning Banach lattices we follow [1] and [5]. Consider a Banach lattice $E$ and a positive weakly compact operator $T: E \rightarrow E$. Now, if $S: E \rightarrow E$ is an operator such that $0 \leqslant S \leqslant T$ holds, then what effect does the weak compactness of $T$ have on $S$ ? Before giving some positive answers to this question, we shall present two examples to show that (in general) under these conditions $S$ need not be a weakly compact operator.

EXAMPLE 1. Let $\left\{r_{n}\right\}$ denote the sequence of Rademacher functions on $[0,1]$. That is, $r_{n}(t)=\operatorname{Sgn} \sin \left(2^{n} \pi t\right)$. Consider $S, T: L_{1}[0,1] \rightarrow l_{\infty}$ defined by

$$
S(f)=\left(\int_{0}^{1} f(x) r_{n}^{+}(x) d x\right) \text { and } T(f)=\left(\int_{0}^{1} f(x) d x, \int_{0}^{1} f(x) d x, \ldots\right) .
$$

Then $T$ is compact (it has rank one), and $0 \leqslant S \leqslant T$ holds. On the other hand, $S$ is not a weakly compact operator. To see this, start by observing that the sequence

$$
u_{n}=(\underbrace{0,0, \ldots, 0}_{n \text { places }}, 1,1, \ldots)
$$

of $l_{\infty}$ has no subsequence that converges weakly to zero. Indeed, if $\left\{w_{n}\right\}$ is a subsequence of $\left\{u_{n}\right\}$, then $\left\|w_{n}\right\|_{\infty}=1$ for each $n$ and $w_{n} \downarrow 0$ hold, which show that $\left\{w_{n}\right\}$ cannot converge weakly to zero [1, Theorem 9.8, $\left.\mathrm{p} 63\right]$.

Now consider the sequence $\left\{f_{k}\right\}$ of $L_{1}[0,1]$ defined by $f_{k}=2^{k} \chi_{\left[0,2^{-k}\right]}$. Clearly, $\left\|f_{k}\right\|_{1}=1$ holds for all $k$. An easy computation shows that

$$
S\left(f_{k}\right)=(\underbrace{1,1, \ldots, 1}_{k \text { places }}, \frac{1}{2}, \frac{1}{2}, \frac{1}{2}, \ldots) .
$$

Received by the editors September 3, 1980; presented to the Society, January 8, 1981. 1980 Mathematics Subject Classification. Primary 46A40, 46B30, 47B05, 47B55.

Key words and phrases. Banach lattice, positive operator, weakly compact operator. 
Also, it is easy to see that the only possible weak limit of any subsequence of $\left\{S\left(f_{k}\right)\right\}$ is $e=(1,1,1, \ldots)$. However, the relation $S\left(f_{k}\right)-e=-\frac{1}{2} u_{k}$ shows that no subsequence of $\left\{S\left(f_{k}\right)\right\}$ can converge weakly. In other words, $S$ is not weakly compact.

Example 2. Let $S$ and $T$ be as they were defined in Example 1. Consider the Banach lattice $E=L_{1}[0,1] \oplus l_{\infty}$. Note that neither $E$ nor $E^{\prime}$ has an order continuous norm. Now define $S_{1}, T_{1}: E \rightarrow E$ via the matrices

$$
S_{1}=\left(\begin{array}{ll}
0 & 0 \\
S & 0
\end{array}\right) \text { and } T_{1}=\left(\begin{array}{ll}
0 & 0 \\
T & 0
\end{array}\right)
$$

Clearly, $0 \leqslant S_{1} \leqslant T_{1}$ holds and $T_{1}$ is a compact operator. On the other hand, it should be obvious that $S_{1}$ is not weakly compact.

For a Banach lattice $E$ we denote its norm dual by $E^{\prime}$ and its order continuous dual by $E_{n}^{\sim}$. If $A$ is an ideal of $E$ and $B$ is an ideal of $E^{\prime}$, then the absolute weak topology $|\sigma|(B, A)$ is the locally convex-solid topology on $B$ generated by the family of Riesz seminorms $\left\{\rho_{x}: x \in A\right\}$, where $\rho_{x}(f)=|f|(|x|)$ for each $f \in B$. For details see [1].

In order to obtain our main result we need a lemma. As usual, $E$ is identified with its canonical image in its double dual $E^{\prime \prime}$.

LEMMA 3. If $E$ is a Banach lattice, then $E$ is $|\sigma|\left(E^{\prime \prime}, E^{\prime}\right)$-dense in the ideal generated by $E$ in $E^{\prime \prime}$.

Proof. Let $I$ be the ideal generated by $E$ in $E^{\prime \prime}$. Clearly, $I \subseteq\left(E^{\prime}\right)_{n}^{\sim}$ holds. By [1, Theorem 19.10, p. 129] we know that $E$ is $|\sigma|\left(\left(E^{\prime}\right)_{n}^{\sim}, E^{\prime}\right)$-dense in $\left(E^{\prime}\right)_{n}^{\sim}$. Thus, in view of $E \subseteq I \subseteq E^{\prime \prime} \cap\left(E^{\prime}\right)_{n}^{\sim}$ and the fact that $|\sigma|\left(\left(E^{\prime}\right)_{n}^{\sim}, E^{\prime}\right)$ and $|\sigma|\left(E^{\prime \prime}, E^{\prime}\right)$ agree on $I$, it follows that $E$ is $|\sigma|\left(E^{\prime \prime}, E^{\prime}\right)$-dense in $I$.

We now come to the main theorem of the paper. The result states that if a positive operator is dominated by a weakly compact operator, then its second power is always a weakly compact operator (although the operator need not be weakly compact).

THEOREM 4. Let $E$ be a Banach lattice and let $T: E \rightarrow E$ be a positive weakly compact operator. If an operator $S: E \rightarrow E$ satisfies $0<S<T$, then $S^{2}$ is a weakly compact operator.

Proof. Let $I$ be the ideal generated by $E$ in $E^{\prime \prime}$. The weak compactness of $T$ is equivalent to $T^{\prime \prime}\left(E^{\prime \prime}\right) \subseteq E$ [3, Theorem 2, p. 482]. Thus, if $0<u \in E^{\prime \prime}$, then in view of $0 \leqslant S^{\prime \prime} \leqslant T^{\prime \prime}$ we have $0 \leqslant S^{\prime \prime}(u) \leqslant T^{\prime \prime}(u) \in E$, so that

$$
S^{\prime \prime}\left(E^{\prime \prime}\right) \subseteq I \text {. }
$$

Next we claim that $S^{\prime \prime}$ also satisfies

$$
S^{\prime \prime}(I) \subseteq E \text {. }
$$

If this has been established, then (1) and (2) give $\left(S^{2}\right)^{\prime \prime}\left(E^{\prime \prime}\right)=S^{\prime \prime}\left[S^{\prime \prime}\left(E^{\prime \prime}\right)\right] \subseteq$ $S^{\prime \prime}(I) \subseteq E$, i.e., that $S^{2}$ is weakly compact.

The rest of the proof is devoted to establishing (2). To this end, let $0<u \in I$. Pick some $x \in E$ with $0 \leqslant u \leqslant x$. By Lemma 3 , there exists a net $\left\{x_{\alpha}\right\}$ of $E$ 
converging to $u$ for $|\sigma|\left(E^{\prime \prime}, E^{\prime}\right)$ (and hence, for $\sigma\left(E^{\prime \prime}, E^{\prime}\right)$ ) and satisfying $0<x_{\alpha}<$ $x$ for all $\alpha$. Let $B^{\prime}$ denote the closed unit ball of $E^{\prime}$. The weak compactness of $T$ implies that $T^{\prime}:\left(E^{\prime}, \sigma\left(E^{\prime}, E\right)\right) \rightarrow\left(E^{\prime}, \sigma\left(E^{\prime}, E^{\prime \prime}\right)\right)$ is continuous [3, Lemma 7, p. 484]. Thus, $T^{\prime}\left(B^{\prime}\right)$ is $\sigma\left(E^{\prime}, E^{\prime \prime}\right)$-compact, and therefore, $T^{\prime}\left(B^{\prime}\right)$ is also compact for the coarser topology $\sigma\left(E^{\prime}, I\right)$.

Now let $\varepsilon>0$. Combining Theorems 20.14 and 20.6 of [1] or by using [2, Theorem 3.2, p. 192] we see that there exists some $0<g \in E^{\prime}$ satisfying

$$
\left\langle\left(\left|T^{\prime} f\right|-g\right)^{+}, x\right\rangle\left\langle\varepsilon \text { for all } f \in B^{\prime} .\right.
$$

Choose $\alpha_{1}$ such that $\left\langle g,\left|x_{\alpha}-x_{\beta}\right|\right\rangle\left\langle\varepsilon\right.$ holds for all $\alpha, \beta>\alpha_{1}$. Then for $\alpha, \beta>\alpha_{1}$ and $f \in B^{\prime}$ we have

$$
\begin{aligned}
\left|\left\langle f,\left|S x_{\alpha}-S x_{\beta}\right|\right\rangle\right| \leqslant\left\langle|f|, S\left|x_{\alpha}-x_{\beta}\right|\right\rangle \leqslant\left\langle|f|, T\left|x_{\alpha}-x_{\beta}\right|\right\rangle \\
=\left\langle T^{\prime}|f|,\left|x_{\alpha}-x_{\beta}\right|\right\rangle \leqslant\left\langle\left(T^{\prime}|f|-g\right)^{+},\left|x_{\alpha}-x_{\beta}\right|\right\rangle+\left\langle g,\left|x_{\alpha}-x_{\beta}\right|\right\rangle \\
\leqslant 2\left\langle\left(T^{\prime}|f|-g\right)^{+}, x\right\rangle+\left\langle g,\left|x_{\alpha}-x_{\beta}\right|\right\rangle\langle 3 \varepsilon .
\end{aligned}
$$

This implies easily $\left\|S x_{\alpha}-S x_{\beta}\right\| \leqslant 3 \varepsilon$ for all $\alpha, \beta>\alpha_{1}$, so that $\left\{S x_{\alpha}\right\}$ is a norm Cauchy net of $E$. If $y$ is its norm limit in $E$, then using the weak* continuity of $S^{\prime \prime}$ we have the following $\sigma\left(E^{\prime \prime}, E^{\prime}\right)$-limits:

$$
S^{\prime \prime}(u)=\lim S^{\prime \prime} x_{\alpha}=\lim S x_{\alpha}=y \in E .
$$

Thus, (2) is established, and the proof of the theorem is complete.

The preceding proof (with minor adjustments) yields, in actuality, the following general version.

Theorem 5. Let $F, G$, and $H$ be Banach lattices, $F \stackrel{S_{1}}{\rightarrow} G \stackrel{S_{2}}{\rightarrow} H$ and $F \stackrel{T_{1}}{\rightarrow} G \stackrel{T_{2}}{\rightarrow} H$ be positive operators such that $0 \leqslant S_{i} \leqslant T_{i}(i=1,2)$ holds. If $T_{1}$ and $T_{2}$ are weakly compact operators, then the composition operator $S_{2} S_{1}$ is likewise a weakly compact operator.

The proof of Theorem 5 can also be obtained directly from Theorem 4 as follows: Consider $E=F \oplus G \oplus H$, and $S, T: E \rightarrow E$ defined via the matrices

$$
S=\left(\begin{array}{ccc}
0 & 0 & 0 \\
S_{1} & 0 & 0 \\
0 & S_{2} & 0
\end{array}\right) \text { and } T=\left[\begin{array}{ccc}
0 & 0 & 0 \\
T_{1} & 0 & 0 \\
0 & T_{2} & 0
\end{array}\right)
$$

Then $0 \leqslant S \leqslant T$ holds, $T$ is weakly compact, and

$$
S^{2}=\left(\begin{array}{ccc}
0 & 0 & 0 \\
0 & 0 & 0 \\
S_{2} S_{1} & 0 & 0
\end{array}\right)
$$

By Theorem $4, S^{2}$ is weakly compact, and so, $S_{2} S_{1}$ is likewise a weakly compact operator.

An immediate application of Theorem 4 is the following.

Corollary 6. Let $E$ be a Dedekind complete Banach lattice and $T: E \rightarrow E$ an order bounded operator. If the modulus $|T|$ of $T$ is weakly compact, then $T^{2}$ is weakly compact. 
So far, it is clear from our discussion that if we wish to conclude from $0 \leqslant S<T$ (and the weak compactness of $T$ ) that $S$ is weakly compact, then some assumptions must be added either to the space or to the operator. The next two results are of this type.

Recall that a Banach lattice is said to have an order continuous norm whenever $u_{\alpha} \downarrow 0$ implies $\left\|u_{\alpha}\right\| \downarrow 0$. Any Banach lattice with an order continuous norm is Dedekind complete; see [1] and [5].

THEOREM 7. Let $E$ and $F$ be two Banach lattices with either $E^{\prime}$ or $F$ having an order continuous norm. Also assume that $S, T: E \rightarrow F$ are two operators satisfying $0 \leqslant S \leqslant T$. If $T$ is weakly compact, then $S$ is likewise weakly compact.

Proof. Assume first that $F$ has an order continuous norm. Then $F$ is an ideal of $F^{\prime \prime}$; see [1, pp. 60-61]. Thus, if $0 \leqslant u \in E^{\prime \prime}$, then the relation $0<S^{\prime \prime} u<T^{\prime \prime} u \in F$ implies $S^{\prime \prime} u \in F$. That is, $S^{\prime \prime}\left(E^{\prime \prime}\right) \subseteq F$ holds, so that $S$ is weakly compact.

Now assume that $E^{\prime}$ has an order continuous norm. In view of $T^{\prime}: F^{\prime} \rightarrow E^{\prime}$ and $0 \leqslant S^{\prime} \leqslant T^{\prime}$ the preceding case shows that $S^{\prime}$ is weakly compact. Therefore, by Gantmacher's theorem, $S$ is likewise weakly compact, and the proof of the theorem is complete.

Note. Theorem 7 was also proved in [8] by a completely different method.

For the next theorem we need the notion of a positive orthomorphism. A positive operator $T: E \rightarrow E$ on a Riesz space $E$ is said to be a positive orthomorphism, whenever $x \wedge y=0$ implies $x \wedge T y=0$. If $E$ is a Banach lattice, then a positive operator $T: E \rightarrow E$ is a positive orthomorphism if and only if there exists some $\alpha>0$ such that $0 \leqslant T \leqslant \alpha I$ holds (where $I$ now denotes the identity operator on $E$ ). See, for example, [7].

THEOREM 8. Let $E$ be a Banach lattice and $T: E \rightarrow E$ a positive weakly compact operator. If a positive orthomorphism $S: E \rightarrow E$ satisfies $0<S \leqslant T$, then $S$ is weakly compact.

Proof. Assume first that $E$ is Dedekind complete. Then by Freudenthal's spectral theorem [4, Theorem 40.2, p. 257], $S$ is the uniform limit (and hence, the norm limit) of a sequence each term of which is a finite sum of the form $\Sigma \alpha_{i} P_{i}$, where each $P_{i}$ is a band projection, each $\alpha_{i} \geqslant 0$, and $0<\Sigma \alpha_{i} P_{i}<S<T$ holds. In view of $0 \leqslant \alpha_{i} P_{i} \leqslant T$ and $P_{i}=P_{i}^{2}$, it follows from Theorem 4 that each $\Sigma \alpha_{i} P_{i}$ is a weakly compact operator. Thus, $S$ is also a weakly compact operator.

The general case can be established by observing that $0<S^{\prime} \leqslant T^{\prime}$ holds, $S^{\prime}$ is a positive orthomorphism on $E^{\prime}, T^{\prime}$ is weakly compact, and $E^{\prime}$ is Dedekind complete.

Now we turn our attention to the following problem. Consider two Banach lattices $E$ and $F$ with $F$ Dedekind complete, and $T: E \rightarrow F$ an order bounded weakly compact operator.

Question. When is the modulus $|T|$ weakly compact?

Conditions under which $|T|$ is weakly compact are desirable. So far, we have been able to obtain an affirmative answer for two very special classes of Banach lattices. 
Recall that a Banach lattice is said to have a Levi norm whenever it follows from $0<x_{\alpha} \uparrow$ and $\sup \left\{\left\|x_{\alpha}\right\|\right\}<\infty$ that $\sup \left\{x_{\alpha}\right\}$ exists; see [1]. If the norm of a Banach lattice $E$ is Levi and order continuous, then $F$ is a band of $F^{\prime \prime}$ [1, Theorem 22.2, p. 159]. Thus, by [5, Theorem 1.5, p. 232] every continuous operator from an abstract $L$-space into a Banach lattice with a Levi order continuous norm is order bounded.

THEOREM 9. Let $E$ be an abstract L-space and $F$ a Banach lattice with a Levi order continuous norm. If an operator $T: E \rightarrow F$ is weakly compact, then its modulus $|T|$ is also weakly compact.

Proof. Let $T: E \rightarrow F$ be weakly compact. We have already mentioned before that $T$ must be order bounded, and so, $|T|$ exists.

Let $B$ denote the closed unit ball of $E$. Then by our hypothesis, $T(B)$ is $\sigma\left(F, F^{\prime}\right)$-relatively compact. Also, from our assumptions it follows that $F=\left(F^{\prime}\right)_{n}^{\sim}$ [1, Theorem 22.2], and hence, $T(B)$ considered as a subset of $\left(F^{\prime}\right)_{n}^{\sim}$ is $\sigma\left(\left(F^{\prime}\right)_{n}^{\sim}, F^{\prime}\right)-$ relatively compact. Now combine Theorems 20.11 and 20.6 of [1] (or use [2, Theorem 2.14, p. 190]) to get that each order bounded disjoint sequence in $F^{\prime}$ converges to zero uniformly on the solid hull of $T(B)$. Next we claim that $|T|(B)$ also has this property. Indeed, if $0 \leqslant f \in F^{\prime} \subseteq F_{n}^{\sim}$ and $x \in B$, then

$$
\begin{aligned}
K f,|T| x\rangle \mid & \leqslant\langle f,|T|(|x|)\rangle=\sup \left\{\left\langle f, \sum_{i=1}^{n}\left|T x_{i}\right|\right\rangle: x_{i} \geqslant 0 \text { and } \sum_{i=1}^{n} x_{i}=|x|\right\} \\
& =\sup \left\{\sum_{i=1}^{n}\left\|x_{i}\right\|\left\langle f,\left|T\left(\frac{x_{i}}{\left\|x_{i}\right\|}\right)\right|\right\rangle: x_{i} \geqslant 0 \text { and } \sum_{i=1}^{n} x_{i}=|x|\right\} \\
& \leqslant \sup \{\langle f,|T y|\rangle: y \in B\} \cdot \sup \left\{\sum_{i=1}^{n}\left\|x_{i}\right\|: x_{i} \geqslant 0 \text { and } \sum_{i=1}^{n} x_{i}=|x|\right\} \\
& \leqslant \sup \{\langle f,|T y|\rangle: y \in B\} .
\end{aligned}
$$

This implies that each order bounded disjoint sequence in $F^{\prime}$ must also converge to zero uniformly on the solid hull of $|T|(B)$.

Now by [1, Theorem 20.6, p. 135], $|T|(B)$ is order-equicontinuous on $F^{\prime}$, and thus, by [1, Theorem 20.11, p. 139] and $F=\left(F^{\prime}\right)_{n}^{\sim},|T|(B)$ is $\sigma\left(F, F^{\prime}\right)$-relatively compact. That is, $|T|$ is a weakly compact operator, and the proof is finished.

The preceding result coupled with Theorem 7 yields the following. ( $\left(\complement_{b}(E, F)\right.$ denotes the Riesz space of all order bounded operators from $E$ into $F$.)

COROLlARY 10. The weakly compact operators from an abstract $L$-space $E$ into a Banach lattice $F$ with a Levi order continuous norm form an ideal of $\varrho_{b}(E, F)$.

When $E$ is a $L$-space and $F$ is a Banach function space on $\sigma$-finite measure spaces, Theorem 9 was proved in [6] by measure theoretical arguments.

Finally, we close the paper by mentioning that our results hold true for locally convex-solid Riesz spaces. Call a continuous operator $T: E \rightarrow F$ between two (Hausdorff) locally convex-solid Riesz spaces weakly compact if it carries topologically bounded subsets of $E$ onto $\sigma\left(F, F^{\prime}\right)$-relatively compact subsets of $F$. For instance, a simple modification of the proof of Theorem 4 yields: Let $E$ be $a$ 
topologically complete (Hausdorff) locally convex-solid Riesz space and let $T: E \rightarrow E$ be a positive weakly compact operator. If an operator $S: E \rightarrow E$ satisfies $0<S<T$, then $S^{2}$ is a weakly compact operator.

\section{REFERENCES}

1. C. D. Aliprantis and O. Burkinshaw, Locally solid Riesz spaces, Academic Press, New York, 1978.

2. O. Burkinshaw, Weak compactness in the order dual of a vector lattice, Trans. Amer. Math. Soc. 187 (1974), 183-201.

3. N. Dunford and J. T. Schwartz, Linear operators. I, Wiley (Interscience), New York, 1958.

4. W. A. J. Luxemburg and A. C. Zaanen, Riesz spaces. I, North-Holland, Amsterdam, 1971.

5. H. H. Schaefer, Banach lattices and positive operators, Springer-Verlag, Berlin and New York and Heidelberg, 1974.

6. A. R. Schep, Compactness properties of an operator which imply that is is an integral operator, Trans. Amer. Math. Soc. 265 (1981), 111-119.

7. A. W. Wickstead, Representation and duality of multiplication operators on Archimedean Riesz spaces, Compositio Math. 35 (1977), 225-238.

8. , Extremal structure of cones of operators, Quart. J. Math. Oxford Ser. (2) 32 (1981), 239-253.

Department of Mathematical Sciences, Indiana University-PuRdue University at IndianapoLIS, INDIANAPOLIS, INDIANA 46205 